\title{
REMARKS ON QUASILINEAR EVOLUTIONS EQUATIONS
}

\author{
JAMES E. MUNOZ RIVERA \\ NATIONAL Laboratory OF SCIENTIFIC COMPUTATION \\ Rua lauro MuLler 455 \\ (LNCC-CNPQ) Botafogo. CEP. 22290. Rio de JaneIRo. R.J. BRagil. \\ AND \\ IMUFRJ. P. O. BOX 68530. RIO de JANEIRO. R.J. BRAsIL \\ (Received June 6, 1990 and in revised form July 21, 1990)
}

ABSTRACT. In this paper we study the existence result of classical
solutions for the quasilinear equation $u_{t t}-\Delta u-M\left(s_{\Omega}|\nabla u|^{2} d \times x^{3} \Delta u_{t t}=f\right.$,
with initial data $u(O)=u_{0}, u_{t}(0)=u_{1}$ and homogeneous boundary conditions.

KEY WORDS.- Partial diferential equation, quasilinear evolution equation, boundary problem.

AMS(MOS): Subject classification. 35B65. 35405.

1. INTRODUCTION: Let $\Omega$ be an open and bounded set of $\mathbb{R}^{n}$, with smooth boundary $\Gamma$. Let's denote by $Q$ the cylinder $\left.Q=\Omega_{x}\right] O . T[$ and by $\Sigma$ its lateral boundary. Our notations and function spaces are standart and follows the same pattern as Lions's book [2].

Ebihara et al [1] was proved that there exist only one classical solution for a semilinear model, given by following initial-boundary value problem

$$
\begin{gathered}
u_{t t}-\Delta u-M\left(\int_{\Omega}|\nabla u|^{2} d x\right) \Delta u_{t t}=f \text { in } Q \\
u(O)=u_{0^{\prime}} u_{t}(O)=u_{1} \text { in } \Omega
\end{gathered}
$$




$$
u(x, t)=0 \text { in } \Sigma
$$

when the following hypotheses hold:

(i) $M(\wedge) \in C^{2}(0,+\infty)$, and there exist positive constants $\alpha$, $p$ such that the following inequality is valid:

$$
M(\lambda) \geq a \sqrt{\lambda}+\rho, \forall \lambda \in(0,+\infty l
$$

(ii) There exists a non negative function $\beta(\lambda)$ satisfying:

$$
\left|\frac{\alpha}{d \lambda} M(\lambda)\right| \sqrt{\lambda} \leq \beta(\lambda) M(\lambda) \quad \forall \lambda \geq 0
$$

(iii) The initial datas are such that:

$$
\begin{gathered}
u_{0^{\prime}} u_{1} \in D\left(A^{\left(t+1: r^{2}\right)}, \quad 1 \geq 1\right. \\
f . \frac{d}{d t} f \in \operatorname{co}\left(T ; D\left(A^{(/ 2}\right)\right), \quad 1 \geq 1
\end{gathered}
$$

Where $A=-\Delta$ and for $D\left(A^{*}\right)$ we are denoting the domain of the operator $A^{=}$. The main result of this paper is to prove the existence result of classical solutions for system (1.1)-(1.3) when

H1. $M$ is a continuos function such that: $M(\lambda) \geq m_{0}>0$

H2. $f \in\left(0, T: D\left(A^{L / 2}\right)\right), L \geq 2$ and $u_{0}, u_{1} \in D\left(A^{(l+1) / 2}\right), 1 \geq 2$

2. THE MAIN RESULT: Let's denote by $w_{1}, \ldots, w_{m}$ and by $\lambda_{1}, \ldots . \lambda_{m}$ the $m$ firts orthonormal eigen functions and eigen values of the Laplacian respectively. Let's denote by $v_{m}$ the finite dimensional vector space generated by the rirts $m$ eigen runctions and by $P_{m}$ the projector operator on $V_{m}$. that is:

$$
P_{m} v=\sum_{i=1}^{m}\left(\int_{\Omega} v(x) w_{L}(x) d x\right) w_{L}
$$

It is easy to see that $A^{s} P_{m}=P_{m} A^{s}$ in $D\left(A^{5}\right)$. Moreover' we have that

$$
\int_{\Omega}\left|P_{m} w\right|^{2} d x \leq \int_{\Omega}|w|^{2} d x
$$

Then the aproximated problem is defined as follows.

$$
\begin{aligned}
& u_{t}^{(m)}-\Delta u^{(m)}-M\left(\int_{\Omega}\left|\nabla u^{(m)}\right|^{2} d x\right) \Delta u_{t t}^{(m)}=f_{m} \\
& u^{(m)}(O)=u_{0^{\prime}}^{m} u_{t}^{(m)}(O)=u_{1}^{m} \text { in } \Omega
\end{aligned}
$$

where

$$
u^{(m)}(t)=\sum_{L=1}^{m} B^{L, m}(t) w_{L}, \quad u_{0}^{m}=P_{m} u_{0}, \quad u_{1}^{m}=P_{m} u_{1}
$$

Before to prove the main result of this paper we will show the following Lemmas:

LEMMA 2.1- Let's suppose that $v, v_{t}, v_{t l} \in C\left(0, T: L^{2}(\Omega)\right.$ and

$$
\int_{\Omega}\left|v_{t t}(x, t)\right|^{2} d x \leq a+b \int_{\Omega}|v(x, t)|^{2} d x
$$


Then we have:

$$
\int_{\Omega}|v(x, t)|^{2} d x \leq\left(a+2 b \int_{\Omega}|v(x, 0)|^{2} d x+4 b t^{2} \int_{\Omega}\left|v_{t}(x, 0)\right|^{2} d x\right) e^{4 b t^{4}}
$$

PROOF.- Since

$$
v(x, t)=\int_{0}^{t} v_{t}(x, y) d \xi+v(x, 0) \text { a. e. in } x
$$

we have:

$$
|v(x, t)| \leq \sqrt{t}\left(\int_{0}^{t}|v,(x, \xi)|^{2} d \xi\right)^{1 / 2}+|v(x, 0)|
$$

From where it follows

$$
\int_{\Omega}|v(x, t)|^{2} d x \leq 2 t \int_{0}^{t} \int_{\Omega}|v,(x, \xi)|^{2} d x d \xi+2 \int_{\Omega}|v(x, 0)|^{2} d x
$$

Applying the relation above to $v_{l}$ we have:

$$
\int_{\Omega}\left|v_{i}(x, t)\right|^{2} d x \leq 2 t \int_{0}^{t} \int_{\Omega}\left|v_{t i}(x, \xi)\right|^{2} d x d \xi+2 \int_{\Omega}\left|v_{\ell}(x, 0)\right|^{2} d x
$$

From the two last inequalities we conclude:

$$
\begin{gathered}
\int_{\Omega}|v(x, t)|^{2} d x \leq \\
2 \int_{\Omega}|v(x, 0)|^{2} d x+4 t^{2} \int_{\Omega}\left|v_{t}(x, 0)\right|^{2} d x+4 t^{3} \int_{0}^{t} \int_{\Omega}\left|v_{t}(x, \xi)\right|^{2} d x d \xi
\end{gathered}
$$

Finally, from the hypotheses, the last inequality and Gronwall's inequality the result of Lemma 2.1 follows a

LEMMA 2.2.- Let suppose that $w \in C(0, T]_{i} L^{2}(\Omega)$, then we have that

$$
P_{m} w \rightarrow w \text { strons in } C\left([0, T]: L^{2}(\Omega)\right.
$$

PROOF.- By the pointwise convergence of $P_{m} w$ in $t$, it's sufficient to show that $P_{m} w$ is a Cauchy sequence in $c\left([0, T]_{i} L^{2}(\Omega)\right)$. Let's take $c>0$, by the continuity of $w$ we have that there exist $\delta>0$ such that

$$
|t-s|<\delta \Rightarrow \int_{\Omega}|w(x, t)-\omega(x, s)|^{2} d x<\frac{\varepsilon}{3}
$$

By the compacity of $[0, T]$, there exist $s_{1}, s_{2}, \ldots s_{N}$, satisrying

$$
\left.[0, T] \subset \bigcup_{1}\right] s_{2}-\delta, s_{1}+\delta l
$$

and from the pointwise convergence of $P_{m} w$ we conclude that there exists a positive number $N$ such that

$$
\left.\int_{\Omega} \mid P_{m} w C \ldots s_{i}\right)-P_{\mu} w C .,\left.s_{i} J\right|^{2} d x<\frac{\varepsilon}{3}, \quad \forall m, \mu \geq N, i=1, \ldots N
$$

Finally by (2.1), (2.3), (2.4) and the following inequality

$$
\begin{aligned}
& {\left[\int_{\Omega}\left|P_{m} w(x, t)-P_{\mu} w(x, t)\right|^{2} d x\right]^{1 / 2} \leq} \\
& {\left[\int_{\Omega}\left|P_{m}\left(\omega(x, t)-\omega\left(x_{0} s_{2}\right)\right\}\right|^{2} d x\right]^{1 / 2}+\left(\int_{\Omega}\left|P_{m} w\left(x_{0} s_{L}\right)-P_{\mu} \omega\left(x_{,} s_{l}\right)\right|^{2} d x\right]^{1 / 2}+} \\
& \left.+\left.\left[\int_{\Omega} \mid P_{\mu}\left(\omega<x_{1} s_{L}\right)-w\left(x_{1} t\right)\right\}\right|^{2} d x\right\}^{1 / 2}
\end{aligned}
$$

the result of Lemma 2.2 follows $\square$

THEOREM 2.3.- Let's suppose that $\mathrm{HI}$ and $\mathrm{HZ}$ are valid. Then there exists 
(1.1), (1.2) and (1.3). Remains to show that $u$ is a classical solution. Let's note that $u^{(m)}$ belongs to $C^{2}\left(0, T: D\left(A^{(l+1) / 2}\right)\right.$ for all $m \in \mathbb{N}$, then in order to prove that $u \in C^{2}\left(O, T_{i} C^{k}(\Omega)\right.$, we will show that $\left(u_{\| l}^{(m)}\right)_{m \in \mathbb{N}}$ is a Cauchy's sequence in $L^{\infty}\left(0, T: D\left(A^{(l+1) / 2}\right)\right)$. for all $l \geq 2$. In fact let $\mu \in \mathbb{N}$, then

$$
\left.u_{t t}^{(\mu)}-\Delta u^{(\mu)}-M \int_{\Omega}\left|\nabla u^{(\mu)}\right|^{2} d x\right) \Delta u_{t t}^{(\mu)}=P_{\mu} f
$$

From (2.2) and the above equation we have:

where

$$
\left.\left(u_{t t}^{(m)}-u_{t t}^{(\mu)}\right)-\Delta\left(u^{(m)}-u^{(\mu)}\right)-M \int_{\Omega}\left|\nabla u^{(m)}\right|^{2} d x\right) \Delta\left(u_{t t}^{(m)}-u_{t t}^{(\mu)}\right)=G_{m \mu}
$$

$$
\left.\left.G_{m \mu}=\left(M \int_{\Omega}\left|\nabla u^{(m)}\right|^{2} d x\right)-M \int_{\Omega}\left|\nabla u^{(\mu)}\right|^{2} d x\right)\right\} \Delta u_{t t}^{(\mu)}+P_{m} f-P_{\mu} f
$$

Multiplying the system above by $A^{l}\left(u_{t l}^{(m)}-u_{t t}^{\langle(\mu)}\right)$ and integrating in $\Omega$ we have

$$
\begin{gathered}
m \int_{\Omega}\left|A^{\frac{l+1}{2}}\left(u_{t t}^{(m)}-u_{t l}^{(\mu)}\right)\right|^{2} d x \leq \\
\int_{\Omega}\left|A\left(u^{(m)}-u^{(\mu)}\right) A^{l}\left(u_{t t}^{(m)}-u_{t t}^{(\mu)}\right)\right| d x+\int_{\Omega}\left|G_{m \mu^{\prime}}\left(u_{t t}^{(m)}-u_{t l}^{(\mu)}\right)\right| d x
\end{gathered}
$$

From which it follows that:

$$
\frac{1}{2} m_{0}^{2} \int_{\Omega}\left|A^{\frac{l+1}{2}}\left(u_{t t}^{(m)}-u_{t t}^{(\mu)}\right)\right|^{2} d x \leq \int_{\Omega}\left|A^{\frac{l+1}{2}}\left(u^{(m)}-u^{(\mu)}\right)\right|^{2} d x+\int_{\Omega}\left|A^{\frac{l}{2}} G_{m \mu}\right|^{2} d x
$$

From Lemma (3.1) and the last inequality we have

$$
\frac{1}{2} m_{0}^{2} \int_{\Omega}\left|A^{\frac{l+1}{2}}\left(u_{t t}^{(m)}-u_{t l}^{(\mu)}\right)\right|^{2} d x \leq
$$

$\left\langle\int_{\Omega}\left|A^{\frac{1}{2}} G_{m \mu}\right|^{2} d x+2 \int_{\Omega}\left|A^{\frac{l+1}{2}}\left(u_{0}^{m}-u_{0}^{\mu}\right)\right|^{2} d x+4 t^{2} \int_{\Omega}\left|A^{\frac{l+1}{2}}\left(u_{1}^{m}-u_{1}^{\mu}\right)\right|^{2} d x\right) E x p\left(\frac{8}{m_{0}^{2}} t^{4}\right)$

Finally from Lemma 2.2 and since $u_{0}, u_{1} \in D\left(A^{(l+1) / 2}\right)$ we have that

$$
A^{L / 2} G_{m \mu} \rightarrow 0 \text { as } m \text {. } \mu \rightarrow+\infty \text { strongly in } c(0, T): L^{2}(\Omega)
$$

Then we have that $\left(u_{t l}^{(m)}\right)$ a Cauchy sequence in $L^{\infty}\left(O, T ; D\left(A^{(l+1) / 2}\right)\right.$ and the proof is now complete 0

REMARK 2.4.- UNIQUENESS: If $M$ is locally Lipschitz, then we have uniqueness. In fact, let $u$ and $v$ be two solutions, putting $w=u-v$ we have

$$
\left.w_{t t}-\Delta w-M\left(\int_{\Omega}|\nabla u|^{2} d x\right) \Delta w_{t t}=\left(M \int_{\Omega}|\nabla u|^{2} d x\right)-M \int_{\Omega}|\nabla v|^{2} d x\right) \Delta v_{t t}
$$

Multiplying by $\Delta w_{t t}$ applying $H 1$ and the Lipschitz condition on $M$ we have that there exists a positive constant $c_{1}$ such that:

$$
m_{0}\left|\Delta w_{\mathrm{tt}}\right|^{2} d x \leq \int_{\Omega}\left|\Delta w \Delta w_{\mathrm{tt}}\right| d x+c_{1}\left(\int_{\Omega}|\Delta w|^{2} d x\right)^{1 / 2}\left(\int_{\Omega}\left|\Delta w_{\mathrm{tt}}\right|^{2} d x\right)^{1 / 2}
$$


only one classic solution of system (1.1), (1.2) and (1.3)

PROOF.- Since $D\left(A^{(l+1) / 2}\right) \subset H^{l+1}(\Omega) \subset C^{k}(\tilde{\Omega})$ if $1+1>\frac{n}{2}+k$, $1 t^{\prime} \cdot s$ surficient to show that there exists a solution of system (1.1). (1.2) and (1.3) satisfying $u \in C^{2}\left([0, T): D\left(A^{(l+1) / 2}\right)\right.$. In order to prove $1 t$ let's multiply (2. 2$)$ by $A^{l} u_{l t}^{(m)}$ and integrating in $\Omega$ we have:

$$
\begin{gathered}
\left.\int_{\Omega}\left|A^{\frac{1}{2}} u_{t l}^{(m)}\right|^{2} d x+M \int_{\Omega}\left|\nabla u^{(m)}\right|^{2} d x\right) \int_{\Omega}\left|A^{\frac{l+1}{2}} u_{t l}^{(m)}\right|^{2} d x= \\
-\int_{\Omega} A u^{(m)} A^{l} u_{t l}^{(m)} d x+\int_{\Omega} f_{m} A^{l} u_{t l}^{(m)} d x
\end{gathered}
$$

By $\mathrm{H} 1$ and $\mathrm{H} 2$ the last equality becomes:

$$
m_{0} \int_{\Omega}\left|A^{\frac{l+1}{2}} u_{t t}^{(m)}\right|^{2} d x \leq \int_{\Omega}\left|A^{\frac{l+1}{2}} u^{(m)} A^{\frac{l+1}{2}} u_{t l}^{(m)}\right| d x+\int_{\Omega}\left|\left[A^{\frac{1}{2}} f_{m}\right] A^{\frac{l}{2}} u_{t L}^{(m)}\right| d x
$$

from where it follows that:

$$
\frac{1}{2} m_{\alpha}^{2} \int_{\Omega}\left|A^{\frac{l+1}{2}} u_{t t}^{(m)}\right|^{2} d x \leq \frac{1}{\lambda_{1}^{2}} \int_{\Omega}\left|\left(A^{\frac{1}{2}} f_{m}\right]\right|^{2} d x+\int_{\Omega}\left|A^{\frac{l+1}{2}} u^{(m)}\right|^{2} d x
$$

By Lemma 2.1 and the above inequality we obtain:

$$
\begin{gathered}
\frac{1}{2} m_{0}^{2} \int_{\Omega}\left|A^{\frac{l+1}{2}} u_{l t}^{(m)}(x, t)\right|^{2} d x \leq \\
\left.i \int_{\Omega}\left|\left[A^{\frac{1}{2}} f_{m}\right]\right|^{2} d x+2 \int_{\Omega}\left|A^{\frac{l+1}{2}} u_{0}^{m}\right|^{2} d x+4 t^{2} \int_{\Omega}\left|A^{\frac{l+1}{2}} u_{1}^{m}\right|^{2} d x\right) E x p\left(\frac{8}{m_{0}^{2}} t^{4}\right)
\end{gathered}
$$

From (2.5) and since:

$$
\begin{aligned}
& \int_{\Omega}\left|A^{\frac{l+1}{2}} u_{t}^{(m)}\left(x_{,} t\right)\right|^{2} d x \leq 2 t \int_{\Omega}\left|A^{\frac{l+1}{2}} u_{t t}^{(m)}(x, t)\right|^{2} d x+2 \int_{\Omega}\left|A^{\frac{l+1}{2}} u_{1}^{m}\right|^{2} d x \\
& \int_{\Omega}\left|A^{\frac{l+1}{2}} u^{(m)}\left(x_{,} t\right)\right|^{2} d x \leq 2 t \int_{\Omega}\left|A^{\frac{l+1}{2}} u_{t}^{(m)}(x, t)\right|^{2} d x+2 \int_{\Omega}\left|A^{\frac{l+1}{2}} u_{0}^{m}\right|^{2} d x
\end{aligned}
$$

we conclude that there exists a subsequence of $\left(u^{(m)}\right)_{m \in N}$, which we still denoting of the same way and a function $u \in L^{\infty}\left(0, T_{i} D\left(A^{(l+1) / 2}\right)\right)$. satisfying

From the last convergences and the Lions-Aubin's theorem (see Lions's [2], theorem 5.1, chap 1) we conclude in particular that:

$$
u^{(m)} \rightarrow u \text { strongiy in } C(0 . T]_{i} H_{0}^{1}(\Omega) \text { as } m \rightarrow \infty
$$

By standard methods we can prove that $u$ is a strong solution of system 
from where it follows that there exists $c_{2}$ such that:

$$
\int_{\Omega}\left|\Delta w_{t t}\right|^{2} d x \leq c_{2} \int_{\Omega}|\Delta w|^{2} d x
$$

By Lemma $\dot{c} .1$, since $w(x, 0)=w_{i}(x, 0)=0$, we obtain that $\Delta w=0$, and from this it follows that $w=0$, that is $u=v 0$

\section{REFERENCES}

1. Y. Ebihara \& D.C. Pereira. - On Global Classical solution of a quasi Iinear hyperbolic equation. Internat. J. Math \& Math. Sci. Vol. 12 No. 1 (1989) 29-38.

2. J. L. Lions. Quelques hodes de resolution de problemes aux limites non lineares. Dunod Gauthier Villars. Paris 1969 


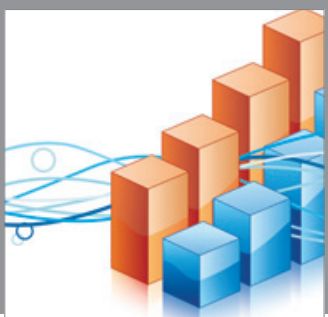

Advances in

Operations Research

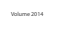

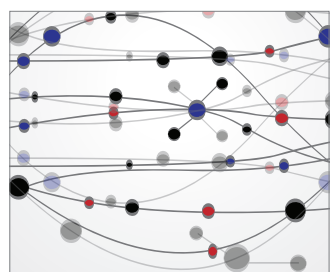

\section{The Scientific} World Journal
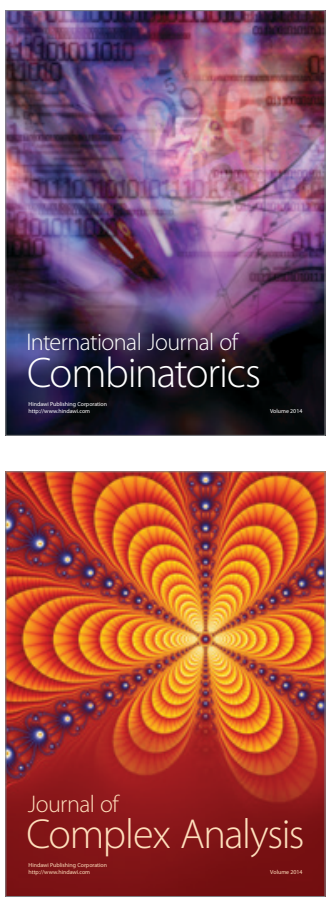

International Journal of

Mathematics and

Mathematical

Sciences
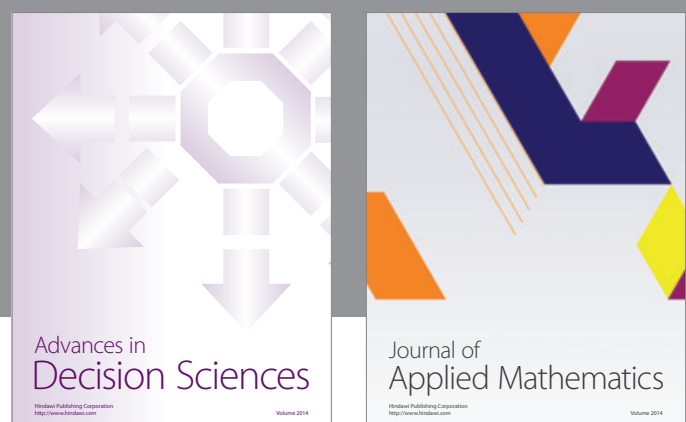

Journal of

Applied Mathematics
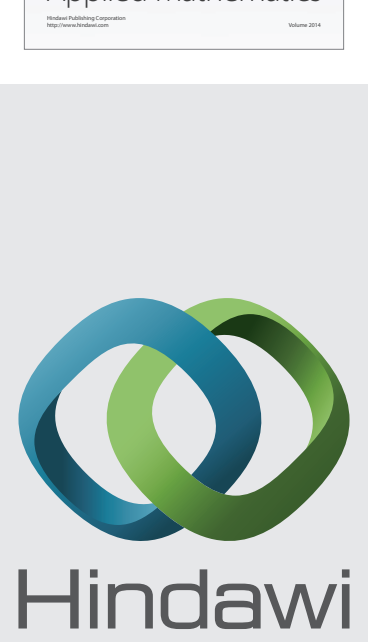

Submit your manuscripts at http://www.hindawi.com
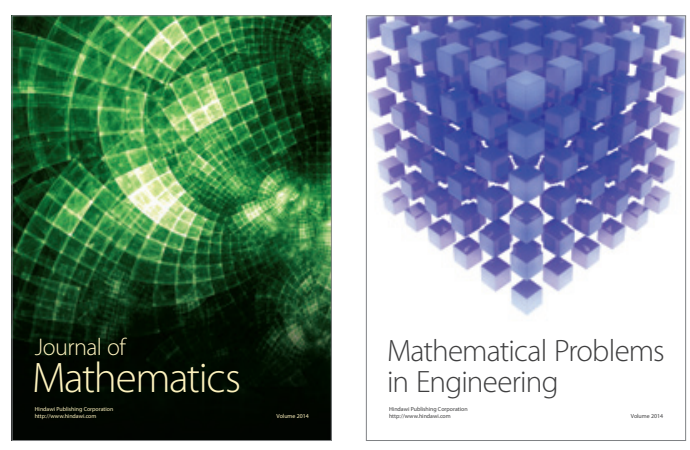

Mathematical Problems in Engineering
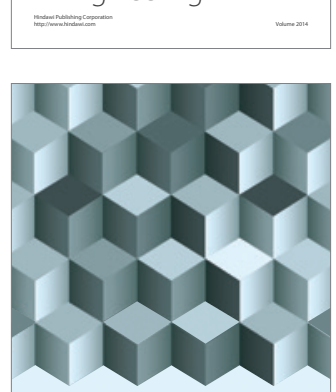

Journal of

Function Spaces
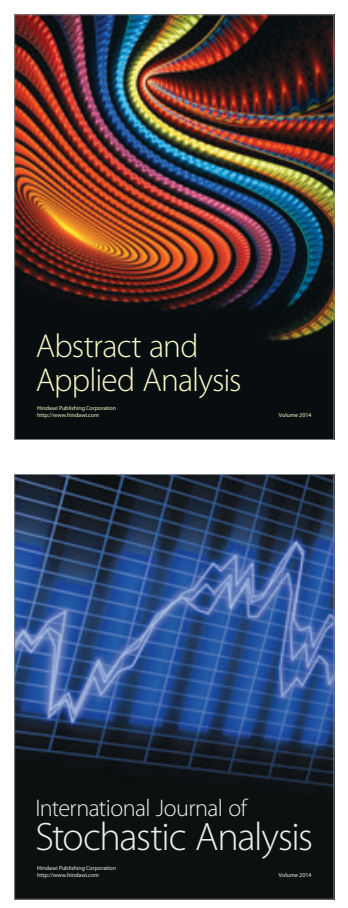

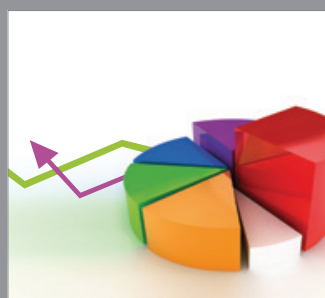

ournal of

Probability and Statistics

Promensencen
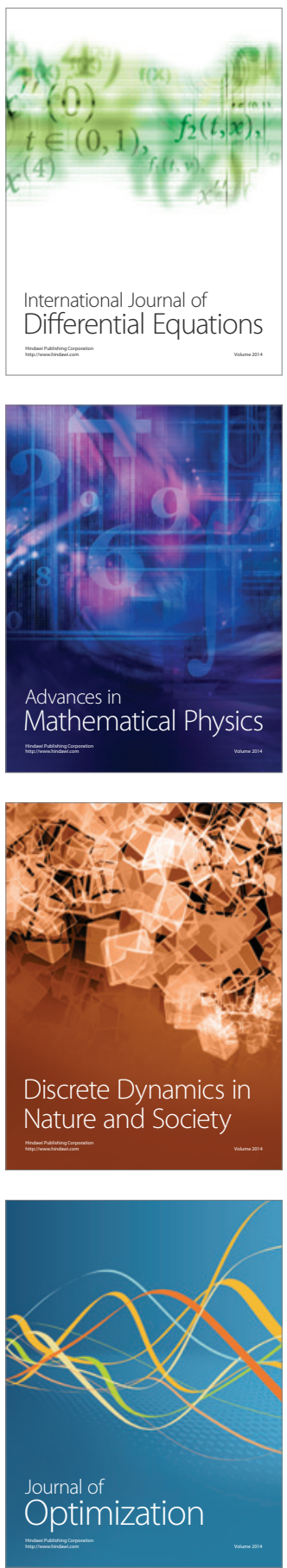